Herz 2016 · 41:459-461

DOI 10.1007/s00059-016-4472-y

Online publiziert: 11. August 2016

(c) Springer Medizin Verlag 2016

CrossMark

\section{B. Maisch}

Philipps-Universität Marburg und Herz- und Gefäßzentrum Marburg, Marburg, Deutschland

\title{
Alkohol und Herz - eine unendliche Geschichte der Wechselbeziehung zur ältesten Droge der Welt
}

\section{Alkohol ist \\ - ein Nahrungsmittel als ein Energie- träger mit erheblichem Kalorienge- halt, \\ - ein gesellschaftlich anerkanntes Ge- nussmittel und damit Bestandteil von zahlreichen, in unserem Kulturkreis gerne genossenen Getränken. Als Kulturgut ist es auch Bestandteil religiöser Rituale, \\ - ein Rauschmittel, \\ - ein Gift, dessen toxische Wirkung sich bei akuter oder chronischer Überdosierung zeigt.}

Alkohol kommt aus dem Arabischen und bezeichnet die aus Antimon bereitete Augenschminke der Ägypter, bedeutet aber auch „etwas Feines“ [1]. Im 16. Jahrhundert übernahm Paracelsus diese Bezeichnung für den Weingeist und nannte ihn Alkohol [2].

\section{Alkoholismus als Krankheit}

Der von Huss im Jahr 1852 eingeführte Begriff Alkoholismus umfasste ursprünglich körperliche Folgeerkrankungen des Alkoholkonsums. Seit 1977 wird, einer der WHO folgenden Empfehlung, zwischen Missbrauch und Abhängigkeit unterschieden [3, 4]. Alkoholmissbrauch verursacht Gesundheitsschädigungen körperlicher oder psychischer Art. Alkoholabhängigkeit beschreibt die physische und psychische Abhängigkeit vom Konsum und die bei Konsumverzicht auftretenden Entzugssymptome [5]. Alkoholabhängigkeit ist die häufigs- te psychische Krankheit bei Männern in den westlichen Industrienationen. Es gibt in Mitteleuropa doppelt so viele männliche wie weibliche Alkoholiker. In Deutschland besteht bei 1,3-1,8 Mio. Menschen eine Alkoholabhängigkeit, bei 2,7 Mio. Menschen ein Alkoholmissbrauch. Alkoholabhängigkeit und -missbrauch kommt in allen sozialen Schichten und allen Altersgruppen in ähnlicher Größenordnung vor [6] .

Alkoholismus bzw. Alkoholsucht ist eine Suchterkrankung. Sie liegt vor, wenn mindestens 3 der folgenden Kriterien nach ICD-10: F10.2 erfüllt sind:

1. Craving: Darunter versteht man ein „Suchtverlangen“ d.h. einen imperativen psychischen Zustand eines Menschen, eine Drogenwirkung wieder erleben zu wollen. Drogen bewirken einen positiv bewerteten Gefühlszustand, der sich mit der Fortdauer der Einnahme abschwächt.

2. Kontrollverlust: d. h. den Verlust der bewussten Steuerung bzw. Beherrschung des Denkens und/oder des Handelns.

3. Körperliches Entzugssyndrom nach Konsumunterbrechung.

4. Toleranzentwicklung.

5. Vernachlässigung anderer Interessen bis zur Verwahrlosung.

6. Fortführen des Konsums trotz klarer Hinweise auf negative körperliche, psychische oder soziale Folgen, z. B. Konsum zu unpassenden Zeiten oder Konsum ohne Rücksicht auf soziale Auswirkungen.
Elvin M. Jellinek führte die in der Medizin auch heute noch gebräuchliche, aber nicht unumstrittene Klassifikation von 5 verschiedenen Typen von „Alkoholikern“ ein [7]. Sie werden mit den ersten 5 Buchstaben des griechischen Alphabets abgekürzt.

Alpha-Trinker (Problemtrinker) trinken, um seelische Belastung leichter zu ertragen. Es besteht keine körperliche Sucht, jedoch eine seelische Abhängigkeit. Das Trinkverhalten ist undiszipliniert. Es kommt jedoch nicht zu einem Kontrollverlust. Gesundheitsschäden und soziale Auffälligkeiten sind nicht selten. Übergang in die Alkoholabhängigkeit vom Gamma-Typ ist häufig.

Beta-Trinker (Gelegenheitstrinker) trinken unter der Übernahme gesellschaftlicher Konsummuster (z. B. auf Feiern jeglicher Art). Obwohl BetaTrinker weder psychisch noch physisch süchtig sind, lassen sie sich leicht zum Konsum verleiten und schädigen durch unverantwortliches Handeln ihre Gesundheit. Beta-Trinker sind suchtgefährdet (nicht selten Übergang in einen Delta-Alkoholismus).

Gamma-Trinker (Suchttrinker) sind psychisch stärker süchtig als physisch. Beim Trinken kommt es zum Kontrollverlust. Trinkexzesse und unauffällige Phasen wechseln sich ab. Durch den ersten Schluck Alkohol wird ein scheinbar unstillbares Verlangen nach immer mehr Alkohol ausgelöst.

Delta-Trinker (Spiegeltrinker) sind körperlich stärker abhängig als psychisch. Delta-Trinker benötigen eine bestimmte Mindestmenge Alkohol, um 
sich gut zu fühlen. Ohne Alkohol leiden Delta-Trinker unter Entzugserscheinungen (Tremor, Schlaflosigkeit usw.) und fallen sozial eher auf.

Epsilon-Trinker (Quartalssäufer) sind psychisch abhängig. Sie können über Monate abstinent sein gefolgt von Episoden exzessiven Alkoholkonsums. In diesen Phasen ist ein Kontrollverlust vorhanden. Trinkexzesse können tagelang fortgeführt werden und zu vorübergehendem Gedächtnisschwund (Filmriss) und illusionärer Verkennung führen. Nach einer solchen Phase folgt in der Regel wieder eine Phase der Abstinenz.

\section{Zahlen}

Der durchschnittliche Pro-Kopf-Verbrauch alkoholischer Getränke in Deutschland liegt bei 137,2 1. Das entspricht 9,7 1 reinen Alkohols. Tatsächlich alkoholabhängig sind ca. 1,3-1,8 Mio. Deutsche. In Deutschland sterben nach Angaben des Drogen- und Suchtberichts $2015 \mathrm{der}$ Bundesregierung rund 74.000 Menschen im Zusammenhang mit Alkoholkonsum. Laut Weltgesundheitsorganisation (WHO) war 2012 bei 3,3 Mio. Todesfällen weltweit Alkohol im Spiel.

Bis zu $12 \mathrm{~g}$ Alkohol bei Frauen (0,14 1 Wein oder 0,31 Bier) und $24 \mathrm{~g}$ bei Männern $(0,251$ Wein oder 0,6 1 Bier) pro Tag werden vom Fachverband Sucht als unbedenklich eingestuft.

9,5 Mio. Menschen in Deutschland konsumieren Alkohol in gesundheitlich riskanter Form. Die volkswirtschaftlichen Kosten belaufen sich auf 26,7 Mrd. Euro, davon sind allein 7,4 Mrd. als direkte Kosten für das Gesundheitssystem $\mathrm{zu}$ veranschlagen.

\section{Genetik und Epigenetik}

Sind Alkoholismus und alkoholische Kardiomyopathie vererbbar?

Die Beziehung zwischen Suchtkrankheiten und Genetik sind komplex. Alkoholismus findet sich gehäuft in Familien, wobei genetische Prädisposition und Einflüsse des persönlichen Umfelds eine etwa gleich starke Rolle spielen [8,9]. Ein Beispiel: Bei vielen Asiaten kommt es wegen eines verlangsamten Abbaus schon bei geringen Mengen von $\mathrm{C}_{2} \mathrm{H}_{5} \mathrm{OH}$ zu unangenehmen Nebenwirkung wie Flushsymptomatik und Tachykardien. Diese Nebenwirkungen wirken abschreckend und damit protektiv. Bei Familienuntersuchungen zeigte sich $z$. B. eine Assoziation des auf Chromosom 11q22q23 codierten Dopaminrezeptors D2 (DRD2) mit dem Copingfaktor für Alkoholismus [10], aber nicht mit dem Serotonintransportergen (SLC6A4), das auf Chromosom 17q11.2 codiert wird. Neben der klassischen Genetik erfährt heute die Epigenetik großes Interesse, die vererbbare Veränderungen der Genexpression durch Alkoholkonsum oder Abstinenz untersucht, die zwar die Genaktivität, nicht aber die Nukleotidsequenz beeinflussen [11].

\section{Geschichten und Geschichte}

Geschichte und Geschichten zur Geschichte haben meist einen Anfang und oft auch ein Ende. In der Beziehung zwischen Alkohol und Menschen im Allgemeinen und Alkohol und Herz im Besonderen gibt es markante Eckpunkte. Der Anfang lag zu Beginn menschlicher Zivilisation und bis heute gibt es kein Ende dieser wechselvollen Beziehung.

Alkohol dürfte vermutlich vor mehr als 12.000 Jahren in der Mittelsteinzeit eher zufällig entdeckt worden sein als Ergebnis des Gärungsprozesses überreifer (Feld-)Früchte. Seit dieser Zeit wird Alkohol als Genussmittel konsumiert. Keilschriftfunde aus Mesopotamien beschreiben den Prozess des Bierbrauens. In den ägyptischen Quellen des 3. Jahrtausends v. Chr. finden sich eindeutige Belege, dass Wein gekeltert und Bier gebraut wurde, und welche Folgen seine narkotisierende Wirkung haben konnte [12].

Als Gott des Weines verehrten die Griechen Dionysos, die Römer Bacchus. Die Germanen stellten aus Honig und Wasser Met her und in Tibet wurde aus Gerste Bier gebraut. Die ersten alkoholischen Erzeugnisse wurden vorwiegend bei kultischen Handlungen konsumiert und blieben wohl einem kleinen Kreis von Würdenträgern, Schamanen und Ältesten vorbehalten. In Ostasien wurden schon früh Weine aus Litschi und Pflaumen hergestellt sowie der Sake, ein warm genossener Reiswein, dessen Herstellungsprozess dem des Bieres ähnelt. In der heutigen Türkei wurde um etwa 1000 n. Chr. die Destillation von Wein zur Herstellung hochprozentiger Branntweine entwickelt.

Haferbier, Cervisa oder Cerevisia genannt, wurde im Mittelalter in Klöstern täglich und in Mengen bis zu $5 \mathrm{Maß}$ konsumiert. Mit der Vergrößerung der landwirtschaftlich nutzbaren Fläche in Amerika im 18. Jahrhundert wurde die Überproduktion an Getreide auch zum Teil zur Herstellung von Gin genutzt und vermehrt getrunken. In der Folge entwickelte sich in England durch den erhöhten Alkoholkonsum die Gin-Krise. Aus dieser Zeit finden sich die ersten Schriften, die sich mit Alkoholabhängigkeit und deren Folgeerkrankungen beschäftigen. Mitte des 19. Jahrhunderts wurde mit der Industrialisierung billiger Alkohol für verarmte Bevölkerungsschichten zugänglich, was sich in Schlagwörtern wie „Branntweinpest" und „Elendsalkoholismus“ widerspiegelte. Im 20. Jahrhundert nahm die industrielle Produktion und Vermarktung, v. a. nach dem Zweiten Weltkrieg, erheblich zu. Alkoholbedingte Schäden zählen heute zu den Zivilisationskrankheiten.

In seinem, mit ausgewählten historischen Details angereicherten Beitrag „Alkohol und Herz - Geschichten zur Geschichte einer wechselvollen Beziehung" führt uns Berndt Lüderitz kenntnisreich und humorvoll von der Antike bis zur Neuzeit durch das Thema, nicht ohne ihm mit Anekdoten Verständnis und Sympathie für die genussreiche Seite des Bier- und Weinkonsums auf seinem Gang durch die Jahrhunderte abzugewinnen.

Ein Plädoyer für Weingenuss und gleichzeitige Prävention bei der koronaren Herzkrankheit geben Markus Flesch, Stephan Morbach, Erland Erdmann und Daniel Bulut. Ihr Resümee lautet: Mäßiger Alkoholkonsum kann bei koronarer Herzerkrankung, bei Angina pectoris und bei Diabetes sogar nützlich sein. Die Beziehung von Alkohol zu kardiovaskulären Erkrankungen verläuft als J-Kurve. Nur starker Konsum von $\mathrm{C}_{2} \mathrm{H}_{5} \mathrm{OH}$ schadet. Mögliche Mechanismen des kardioprotektiven, 
antiatherosklerotischen Effekts mäßigen Alkoholkonsums sind eine Hemmung der Thrombozytenaggregation, eine Erhöhung des HDL-Plasmaspiegels und eine Prävention des Diabetes mellitus.

Eric Leibing und Thomas Meyer zeigen Enzyme und Signaltransduktionswege in der Detoxifikation von Alkohol durch Alkoholdehydrogenase (ADH), Aldehyddehydrogenase (ALDH) und protektive Einflüsse über den STAT3Transkriptionsfaktor mit großer Detailkenntnis und Expertise in ihrem Beitrag „Enzymes and signal pathways involved in the pathogenesis of alcoholic cardiomyopathy" auf.

Im Beitrag „Alcoholic cardiomyopathy - The result of dosage and individual predisposition" sind für Bernhard Maisch historische Beispiele das Münchener Bier- und das Tübinger Weinherz. Nicht auf die reine Alkoholwirkung zurückzuführen sind, aufgrund ihrer andersartigen Ätiologie, das Beriberi-Herz bei Thiamin(Vitamin-B1)-Mangel sowie die kardial mitbedingte Leberzirrhose als hyperdyname Kardiomyopathien. Die als „Manchester Biertrinkerepidemie“ bekannte chronische Herzinsuffizienz war Folge einer Arsenbeimischung beim Filterprozess des Bieres. Die nach Quebec benannte Bierherzerkrankung wurde durch einen Schaumstabilisator verursacht, der zu viel Kobalt enthielt. Chronischer Alkoholabusus ist unmittelbar toxisch für die Herzmuskelzellen. Er erhöht den Blutdruck und belastet dadurch Herz und Gefäße. Er verursacht eine Downregulation des Immunsystems, die ihrerseits zu erhöhter Infektanfälligkeit und zur Myokarditis führen kann. Histologisch ist die alkoholische dann nicht von der idiopathischen Kardiomyopathie, oder bei begleitender Low-grade-Inflammation von einer chronischen Myokarditis zu unterscheiden. Die Abstinenz von der Noxe Alkohol und deren Stützung durch Medikamente wie Naltrexon, Acamprosat oder Disulfiram ist entscheidend für die Prognose, denn daneben bleibt nur die Behandlung mit den bekannten Herzinsuffizienzmedikamenten.

Bei Frauen treten die ungünstigeren Alkoholwirkungen bereits bei wesentlich niedrigeren akut oder chronisch konsu- mierten Mengen auf als bei Männern. Bei Senioren ebenso wie bei Jüngeren zeigt sich, dass ein moderater Alkoholkonsum mit einem niedrigeren Risiko für eine Herzinsuffizienz assoziiert ist. Das sind die Kernpunkte der Übersicht von Sabine Pankuweit in ihrem Beitrag zur Beantwortung der Frage: ,Wann führt Alkoholkonsum bei Frauen und Senioren zur Herzinsuffizienz?"

In ihrem Beitrag zu Alkohol und Rhythmusstörungen kommen Dietrich Pfeiffer, Daniel Jurisch, Martin Neef und Andreas Hagendorff $\mathrm{zu}$ folgenden Feststellungen: Die Auswirkungen von Alkohol auf Arrhythmien sind dosisabhängig, aber unabhängig vom Nachweis einer kardiovaskulären Erkrankung. Sie können Gesunde gleichermaßen betreffen. Während Vorhofflimmern in seiner Wahrscheinlichkeit mit der Alkoholdosis zunimmt, kommen Ereignisse des plötzlichen Herztodes bei niedrigen Alkoholdosierungen eher seltener vor, häufen sich jedoch bei exzessiven Alkoholmengen und bei alkoholischer Kardiomyopathie.

Mein Resümee ist einfach und nicht neu: Alkohol ist Genussmittel und gesellschaftliches Medium einerseits, eine Noxe und ein Rauschmittel andererseits. Darüber entscheiden wir als Konsumenten. Dazu beraten wir aber auch als Ärzte unsere Patienten.

Da kann man Otto von Bollinger bei der Erstbeschreibung des Münchner Bierherzens vor dem ärztlichen Verein in München nur beipflichten:

Während bei mäßigem Genuss vom hygienischen Standpunkt Bier unerreichbare Vorzüge besitzt, ist der Abusus, wie auch bei anderen Spirituosen, gesundheitlich von größten Gefahren begleitet [13].

Die „unendliche Geschichte" der Wechselbeziehung zwischen dem menschlichen Herz und der ältesten Droge der Welt mag zwar immer wieder neu geschrieben werden, aber die Botschaft bleibt mit kleinen Variationen dieselbe:

Regelmäßig wenig Alkohol genießen ist besser als selten viel oder in große Mengen viel zu oft, denn die Dosis und die individuelle Prädisposition machen den Unterschied!

\section{Korrespondenzadresse}

\section{Prof. Dr. B. Maisch}

Philipps-Universität Marburg und Herz- und Gefäßzentrum Marburg

Feldbergstr. 45, 35043 Marburg, Deutschland bermaisch@gmail.com

Interessenkonflikt. B. Maisch gibt an, dass kein Interessenkonflikt besteht.

\section{Literatur}

1. http://www.alkohol-lexikon.de/ALCOHOL/AL GE/geschichte.shtml. Zugegriffen: 8. Aug 2016

2. Maisch B (1996) Alkohol und Herz. Herz21:207-212

3. Feuerlein W (1996) Alkoholismus als Krankheit. Herz 21:214-216

4. Madden JS (1993) The definition of alcoholism. Alcohol 28:617-620

5. Seitz HK, Lesch OM, Spannagel R et al (2013) Alkoholabhängigkeit. Suchtmedizinische Reihe Band 1,Hrsg. DeutscheHauptstelle fürSuchtfragen e.V. http://www.dhs.de/fileadmin/user_upload/ pdf/Broschueren/Suchtmed_Reihe_1_Alkohol. pdf.Zugegriffen: 8. Aug 2016

6. http://www.bptk.de/patienten/psychischekrankheiten/alkoholabhaengigkeit.html. Zugegriffen: 8. Aug 2016

7. Jellinek EM (1960) The disease concept of alcoholism. College and University Press, New Haven and Hillhouse Press

8. Bierut LJ, Dinwiddie SH, Begleiter H et al (1998) Familial transmission of substance dependence: alcohol, marijuana, cocaine, and habitual smoking A report from the collaborative study on the genetics of alcoholism. Arch Gen Psychiatry 55:982-998

9. Goldman D, Oroszi G, Ducc F (2005) The genetics of addictions: uncovering the genes. Nat Rev Genet 6:521-532

10. van der Zwaluw CS, Kuntsche E, Engels RC (2011) Risky alcohol use in adolescence: the role of genetics (DRD2, SLC6A4) and coping motives. Alcohol Clin Exp Res 35(4):756-764

11. Nieratschker V, Batra A, Fallgatter AJ (2013) Genetics and epigenetics of alcohol dependence. JMol Psychiatry 1(1):11

12. Brunold R. Geschichte des Alkohols von der Antike bis zur Weimarer Republik. http://www. geschichte-lernen.net/geschichte-des-alkoholsantike-bis-weimarer-republik. Zugegriffen: 8. Aug 2016

13. von Bollinger O (1884) Über die Häufigkeit und Ursachen der idiopathischen Herzhypertrophie in München.Dtsch Med Wochenschr 10:180 